\title{
Discrete-Log-Based Additively Homomorphic Encryption and Secure WSN Data Aggregation
}

\author{
Licheng Wang ${ }^{1,2}$, Lihua Wang ${ }^{2}$, Yun $\operatorname{Pan}^{3}$, Zonghua Zhang ${ }^{2}$, and Yixian Yang ${ }^{1}$ \\ 1 Information Security Center, State Key Laboratory of Networking and \\ Switching Technology, Beijing University of Posts and Telecommunications \\ 10 West Tucheng Road, Beijing, P.R. China 100876 \\ 2 Security Fundamental Group, Information Security Research Center \\ National Institute of Information and Communications Technology \\ 4-2-1 Nukui-Kitamachi, Koganei-shi, Tokyo, 184-8795 Japan \\ 3 School of Computer, Communication University of China \\ 1 East Street of Dingfuzhuang, Beijing, P.R. China 100024 \\ \{wanglc, wlh, zonghua\}@nict.go.jp, pany@cuc.edu.cn, \\ \{wanglc, yxy\}@bupt.edu.cn
}

\begin{abstract}
At PKC 2006, Chevallier-Mames, Paillier, and Pointcheval proposed encryption schemes that are partially homomorphic, either additively or multiplicatively and announced an open research problem: finding a discrete-log-based cryptosystem that would help realize fully additive or multiplicative homomorphism. In this study, we achieve this goal by lifting the message space of the ElGamal scheme from $\mathcal{M}$ to $g_{0}^{\mathcal{M}}$. We then apply our scheme for constructing a novel protocol for secure data aggregation in Wireless Sensor Networks.
\end{abstract}

Keywords: Discrete-logarithm problem, additively homomorphic encryption, wireless sensor networks, data aggregation.

\section{Introduction}

\subsection{Background: Homomorphic Encryption}

In general, we expect a cryptosystem to be as secure as possible. To this end, various security notions have been developed. The basic requirement for a cryptosystem is that adversaries must be prevented from learning confidential messages. This is the so-called security notion of one-wayness (OW) and was recognized even before 2500 B.C. With the development of modern cryptography, particularly after the advent of public-key cryptosystems [7, new desirable security notions were conceived. Naor suggested that different security notions for encryption should be defined by orthogonally considering the various possible goals and the various possible attack models [1. Typically, two goals, namely, indistinguishability (IND) [13] and non-malleability (NM) [8], and three attack models, namely, chosen-plaintext attack (CPA), non-adaptive chosen-ciphertext attack (CCA1) [14, and adaptive chosen-ciphertext attack (CCA2) [16], have 
been considered. Currently, IND-CCA, especially IND-CCA2, is known to be the most desirable security property for many applied cryptosystems.

However, people also visualized a lot of scenarios in which IND-CCA is too rigid to be flexible. It might be possible to perform some positive transformations on a ciphertext without decryption. Thus, the idea of homomorphic encryption was proposed. Homomorphic encryption enables blind transformations on plaintexts via (possibly different) algebraic operations on ciphertexts 6 4]. Depending upon the specific viewpoint, this is either a positive or a negative attribute of a cryptosystem. Homomorphic encryption schemes are inherently malleable and are thus unsuitable in scenarios in which modifications to ciphertexts are forbidden. On the other hand, the homomorphic property is useful for building secure voting systems, collision-resistant hash functions, private information retrieval schemes, etc. At PKC 2006, Chevallier-Mames, Paillier, and Pointcheval 4 proposed encryption schemes that are partially homomorphic, either additively or multiplicatively and finally announced an open research problem: finding a discrete-log-based cryptosystem that helps realize fully additive or multiplicative homomorphism. Note that there are many additively homomorphic encryption schemes based on factoring-related assumptions, such as Goldwasser-Micali's scheme [12, Benaloh's scheme 2], and Pailliar's scheme 15. Most recently, Gentry proposed a lattice-based fully homomorphic encryption scheme [11].

\subsection{Background II: Secure WSN Data Aggregation}

A very typical application of homomorphic encryption in practice is data aggregation in wireless sensor networks (WSN) [1952013. Aggregation techniques are used to reduce the amount of data communicated within a WSN so as to conserve battery power. Since measurements are recorded by individual sensors, they need to be periodically collected and processed to yield data representative of the entire WSN, such as the average and/or variance of the temperature or humidity within an area [3]. One natural approach to data aggregation is to simply add up values as they are forwarded toward the sink. Homomorphic encryption allows some types of statistical computations on encrypted data, similar to those on plaintext data [10, thereby offering significant advantages in securing WSN data aggregation by preventing eavesdropping attacks. If homomorphic encryption is not used, the intermediate sensors must have access to the secret keys in order to decrypt the collected data before they are added. However, we know that methods in which sensitive information such as secret keys is stored inside sensors are prone to many attacks 3 . Once the secret keys are exposed, the network is no longer secure. Homomorphic encryption prevents this problem since sensitive information is not stored on the intermediate sensors [3].

While it is intuitive to use homomorphic encryption for secure data aggregation in WSN, no silver bullet application has been seen so far, and the majority of available schemes are vulnerable to some extent. For example, in [3], an additively homomorphic encryption for WSN data aggregation was proposed. The scheme is essentially a stream cipher and its homomorphic property relies on the synchronization among the key-stream generators, i.e., all sensors in the 
field must share the same key-stream generator. Clearly, this is impractical in a distributed environment. More seriously, this approach has two fundamental flaws: (1) it is difficult to ensure the confidentiality of this commonly shared keystream generator and (2) the scheme would be insecure if some sensors that have access to the key-stream generator were colluded by the adversaries. Therefore, to achieve secure WSN data aggregation, we prefer homomorphic public-key encryption (Hom-PKE) than secret-key encryption. In public-key encryption, no secret is embedded in the sensors, and thus, this scheme is immune to colluding attacks. Vaidehi [10 tried to solve this problem by using a privacy homomorphism called DF-a-New-PH. However, we found that DF-a-New-PH does not have the desired effect of encryption for two reasons: (1) some secret parameters can be easily computed from the public parameters and (2) all secret parameters are explicitly involved in the encryption algorithm. We know that no public-key encryption scheme can operate in this manner. DF-a-New-PH is essentially an encoding/decoding system rather than a cryptosystem since it does not provide confidentiality. In DF-a-New-PH, all secrets can be made public! Of course, when using Hom-PKE, a malicious node can still insert junk data to disturb the aggregation. However, this problem is not specific to Hom-PKE. All encryption schemes, public-key or secret-key, are vulnerable to this kind of attack. In terms of consequence, this kind of attack can be viewed as denial of service (DOS) attack. How to resist DOS or DDOS attacks is another significant research topic that is beyond the scope of this paper.

\subsection{Motivation and Contributions}

In this paper, we first try to answer the open problem raised by Chevallier-Mames, Paillier, and Pointcheval 4. We achieve this goal by lifting the message space in the ElGamal scheme. This yields a discrete-logarithm-based additively homomorphic encryption scheme. Then, we design a new protocol for WSN data aggregation by employing the proposed additively homomorphic encryption scheme.

\section{Intractability of Discrete Logarithm Problems}

Definition 1 (Discrete Logarithm Problem, DLP). Let $G$ be a finite cyclic group of order $n$ and $g$ be a generator. For any $h \in G$, the discrete logarithm of $h$ with respect to $g$, denoted $D L O G_{g}(h)$, is the element $x \in \mathbb{Z}_{n}$ such that $h=g^{x}$.

In particular, in the remain sections we assume that $G=\mathbb{Z}_{p}^{*}$, where $p$ is a large prime. The security of the Diffie-Hellman protocol [7], as well as ElGamal encryption scheme [9] rely on the intractability of DLP over $\mathbb{Z}_{p}^{*}$ for secure prime $p$. Note that not all discrete logarithm problems are intractable. In fact, the difficulty of computing discrete logarithms in $\mathbb{Z}_{p}^{*}$ is determined by the size of the largest prime factor of $p-1$. More specifically, given the prime factoring $p-1=\prod_{i=1}^{r} q_{i}^{e_{i}}$ and $q=\max \left\{q_{1}, \cdots, q_{r}\right\}$, the running time for computing $x$ is bounded by $q^{1 / 2} \operatorname{len}(p)^{\mathcal{O}(1)}[18$, where $\operatorname{len}(\cdot)$ indicates the bit-length of a given number. This suggests that when $p-1$ does not contain any large prime factor, 
the discrete logarithm problem over $\mathbb{Z}_{p}^{*}$ can be solved efficiently. A prime $p$ is called secure prime if $p-1$ contains large, say at least 160 bits, prime factors.

In subsequent contexts, we assume that $q, p=2 q+1$ are large primes and $G=<g>\subset \mathbb{Z}_{p}^{*}$ be a cyclic group of order $q$ generated by $g$. Other frequently used DLOG-based cryptographic problems are

Definition 2 (Computational Diffie-Hellman problem, CDH). Given $g^{x}$, $g^{y} \in G$ for some unknown $x, y \in \mathbb{Z}_{q}$, compute $g^{x y} \in G$.

Definition 3 (Decisional Diffie-Hellman problem, DDH). Given two distributions $D=\left(g^{x}, g^{y}, g^{x y}\right) \in G^{3}$ and $R=\left(g^{x}, g^{y}, g^{z}\right) \in G^{3}$ for randomly distributed $x, y, z \in \mathbb{Z}_{q}$, distinguish $D$ from $R$.

It is easily seen that $D D H \preceq_{P} C D H \preceq_{P} D L P$ where $\preceq_{P}$ denotes polynomial reduction between computational problems. At present, we know that for a secure prime $p, \mathrm{DLP}, \mathrm{CDH}$, and DDH are intractable, except for resorting a quantum computer 17 .

\section{The Proposed Scheme}

\subsection{OW-CPA ElGamal and Multiplicative Homomorphism}

Recall that the original version of the ElGamal cryptosystem [9] is multiplicatively homomorphic, and thus, let us denote it by ${ }^{\times}$HomElG. The encryption algorithm in ${ }^{\times}$HomElG is denoted by $\mathcal{E}_{\times}$and is multiplicatively homomorphic in the sense that under the same public key $y=g^{x} \bmod p$ (where $g$ is a generator of some $q$ order subgroup of $\mathbb{Z}_{p}^{*}$ with $q \mid p-1$, both $p$ and $q$ are large primes and $x$ is the private key picked randomly from $\mathbb{Z}_{q}$ ), given two ciphertexts

$$
\begin{aligned}
& \mathcal{E}_{\times}\left(y ; m_{1}\right)=\left(g^{r_{1}} \bmod p, y^{r_{1}} m_{1} \bmod p\right), \text { and } \\
& \mathcal{E}_{\times}\left(y ; m_{2}\right)=\left(g^{r_{2}} \bmod p, y^{r_{2}} m_{2} \bmod p\right),
\end{aligned}
$$

anyone can, without knowing the secret key $x$, the original messages $m_{i}$, and the random numbers $r_{i}(i=1,2)$, compute the ciphertext for the plaintext $m_{1} m_{2}$ by using the following formula:

$$
\mathcal{E}_{\times}\left(y ; m_{1} m_{2}\right)=\left(g^{r_{1}} \cdot g^{r_{2}} \bmod p, y^{r_{1}} m_{1} \cdot y^{r_{2}} m_{2} \bmod p\right) .
$$

More formally, given two valid ciphertexts $\left(c_{11}, c_{12}\right)$ and $\left(c_{21}, c_{22}\right)$ for the messages $m_{1}$ and $m_{2}$ under a common public key, $\left(c_{11}, c_{12}\right) \odot\left(c_{21}, c_{22}\right)$ would be a valid ciphertext for the message $m_{1} \cdot m_{2}$ under the same public key, where $\odot$ denotes component-wise modular multiplication, i.e.,

$$
\left(c_{11}, c_{12}\right) \odot\left(c_{21}, c_{22}\right)=\left(c_{11} \cdot c_{21} \bmod p, c_{12} \cdot c_{22} \bmod p\right) .
$$

Note that this version of ElGamal merely achieves OW-CPA security, i.e., onewayness against chosen plaintext attacks. 


\subsection{Message Lifting and Parameter Selection}

A natural idea for converting a multiplicatively homomorphic scheme into an additively homomorphic one is to lift the message space $\mathcal{M}$ to the exponential space $g_{0}^{\mathcal{M}}$ for some base $g_{0}$. To maintain the compactness of the lifted message space, we choose a prime $p_{0}$ such that $p_{0}-1$ does not contain large prime factors and then define the following lifting operation:

$$
m \mapsto g_{0}^{m} \bmod p_{0}, \forall m \in\left\{0, \cdots, p_{0}-2\right\},
$$

where $g_{0}$ is a primitive root of $\mathbb{Z}_{p_{0}}^{*}$. Further, let $L_{g_{0}}(\cdot)$ indicate the reverse, i.e., an unlift operation that is defined by

$$
L_{g_{0}}\left(g_{0}^{m} \bmod p_{0}\right)=m \bmod \left(p_{0}-1\right) .
$$

Note that the precision is determined by the size of the encoding space. Thus, the precision increases with the prime $p_{0}$. Typically, we can choose $p_{0}=2^{16}+1$ and $g_{0}=3$. Then, the sensed data are 16 bits long and the sensing precision is $\frac{d_{\max }-d_{\min }}{2^{16}}$, where $d_{\max }$ and $d_{\min }$ are potential maximum and minimum of the concerned data. This is perhaps suitable for most WSN-oriented applications in which the relevant data would be temperature, humidity, atmospheric pressure, noise, etc.

\subsection{DLP-Based Additive Homomorphism}

Now, let us proceed to develop an additively homomorphic ElGamal encryption scheme, denoted ${ }^{+}$HomElG. This scheme is based on the original multiplicatively homomorphic ElGamal scheme, ${ }^{\times}$HomElG.

- Key-generation algorithm $\mathcal{K}\left(1^{k}\right)$ : Take as input the security parameter $k$, choose two primes $p$ and $p_{0}$ such that

(1) $p=2 q+1$ for some large prime $q$,

(2) $p_{0}=2 t^{\kappa}+1<p$ for a small prime $t$ and some positive integer $\kappa$.

And then, select two generators $g$ and $g_{0}$ so that $g$ generates a $q$-order subgroup of $\mathbb{Z}_{p}^{*}$, while $g_{0}$ generates the group $\mathbb{Z}_{p_{0}}^{*}$. (Typically, let $t=2, \kappa=15$ and $g_{0}=3$.) Next, randomly select a private key $x$ from $\mathbb{Z}_{q}$ and compute the corresponding public key $y=g^{x} \bmod p$. Finally, output the system public parameters

$$
\text { params }=\left(p, q, g ; p_{0}, g_{0}\right)
$$

and return the public/private key pair $(y, x)$. Note that the message space is $\mathcal{M}=\left\{0, \cdots, p_{0}-2\right\} \subset \mathbb{Z}_{p}^{*}$, while the ciphertext space is $\mathcal{C}=\mathbb{Z}_{p}^{*} \times \mathbb{Z}_{p}^{*}$. The security of the scheme is rooted in the secure prime $p$ and irrelevant to $p_{0}$.

Remark 1. Under this condition, we have that: (1) $\left|\mathbb{Z}_{p_{0}}^{*}\right|=p_{0}-1$ and thus $\left(g_{0}^{m} \bmod p_{0}\right)$ goes through the set $\left\{1, \cdots, p_{0}-1\right\}$ when $m$ goes through the set $\left\{0, \cdots, p_{0}-2\right\}$; and (2) the maximal prime factor of $\left(p_{0}-1\right)$ is $t$, and thus the unlifing operation can be finished efficiently. Note that our scheme also works for other form prime $p_{0}$ when $p_{0}-1$ does not contain large prime 
factors. But for simplicity, we merely select $p_{0}$ taking the form $p_{0}=2 t^{\kappa}+1$ for some small prime $t$ and some positive integer $\kappa$. Then, the time complexity of unlifting operation is bounded by $\sqrt{2} \log ^{c} p_{0}$ for some constant $c$ [18].

- Encryption algorithm $\mathcal{E}_{+}(y ; m)$ : Take as inputs the public key $y$ and a message $m \in \mathcal{M}$, choose $r \in \mathbb{Z}_{q}$ at random and output the ciphertext $\left(c_{1}, c_{2}\right)$, where

$$
c_{1}=g^{r} \bmod p, \quad c_{2}=y^{r} \cdot\left(g_{0}^{m} \bmod p_{0}\right) \bmod p .
$$

- Decryption algorithm $\mathcal{D}_{+}\left(x ;\left(c_{1}, c_{2}\right)\right)$ : Take as inputs the secret key $x$ and a ciphertext $\left(c_{1}, c_{2}\right)$, compute the plaintext $m$ by using the decryption algorithm of ${ }^{\times} \mathrm{HomElG}$ and then perform unlifting, i.e.,

$$
m=L_{g_{0}}\left(\mathcal{D}_{\times}\left(x ;\left(c_{1}, c_{2}\right)\right)\right),
$$

where $\mathcal{D}_{\times}$is defined by

$$
\mathcal{D}_{\times}\left(x ;\left(c_{1}, c_{2}\right)\right)=c_{2} / c_{1}^{x} \bmod p .
$$

Remark 2. In practice, we need pay attention to the issue of overflow 1 . Notice that when $\left(g_{0}^{m} \bmod p_{0}\right)^{N}>p$, the following inequality

$$
\left(\left(g_{0}^{m} \bmod p_{0}\right)^{N} \bmod p\right) \bmod p_{0} \neq\left(\left(g_{0}^{m \cdot N} \bmod p_{0}-1 \bmod p_{0}\right) \bmod p\right) \bmod p_{0}
$$

might hold. This suggests the ciphertext on the message $N \cdot m$ cannot be correctly formed by using the proposed scheme. Considering there exists $m^{*}$ such that $g_{0}^{m^{*}} \bmod p_{0}$ achieves the maximum, $p_{0}-1$. In order to compute the ciphertext of $N \cdot m^{*}$ correctly by using the above additive homomorphism, $N$ should satisfy $N<\log p / \log \left(p_{0}-1\right)$, where $N$ indicates the maximal number of the messages than can be correctly added up. This undesirable restrictions might bring inconvenient for certain applications. For example, if $p$ and $p_{0}$ are 1024-bit and 16-bit primes as aforementioned, $N \leq 64$.

The following theorem that captures the consistency and the security of the proposed scheme.

Theorem 1. Under the same public key $y$ and for $\forall m_{1}, m_{2} \in \mathcal{M}$, we have that

$$
\mathcal{E}_{+}\left(y ; m_{1}\right) \odot \mathcal{E}_{+}\left(y ; m_{2}\right)=\mathcal{E}_{+}\left(y ; m_{1}+m_{2}\right),
$$

and under the same private key $x$ and for $\forall\left(c_{11}, c_{12}\right),\left(c_{21}, c_{22}\right) \in \mathcal{C}$, we have that $\mathcal{D}_{+}\left(x ;\left(c_{11}, c_{12}\right)\right)+\mathcal{D}_{+}\left(x ;\left(c_{21}, c_{22}\right)\right) \equiv \mathcal{D}_{+}\left(x ;\left(c_{11}, c_{12}\right) \odot\left(c_{21}, c_{22}\right)\right) \quad\left(\bmod p_{0}-1\right)$.

Proof. See Appendix A.

Theorem 2. The proposed cryptosystem is indistinguishable against chosen plaintext attack assuming that the decisional Diffie-Hellman problem over $\mathbb{Z}_{p}^{*}$ (where $p$ is a secure prime) is intractable.

Proof. Omitted for space limitation.

${ }^{1}$ This issue is noticed by an anonymous referee (cf. Acknowledgments). 


\section{Protocol for Secure WSN Data Aggregation}

Without loss of generality, let us visualize a multilevel network tree in which numerous sensor nodes and only one sink node exist. We assume that all nodes are potential aggregators and that data get aggregated as they propagate toward the sink. Suppose the system public parameters, as well as the sink's public key $y$, are input to each sensor node beforehand. Now, the protocol for secure WSN data aggregation involves the following steps:

- Parameters selection. Suppose that the maximum number of potential sensor nodes is at most $N 2$ the range of the sensed data is $\left[d_{\min }, d_{\max }\right]$, and the desired sensing precision is $\delta$. Then, we should select prime $p_{0}$ so that $p_{0}-1>$ $N \frac{d_{\max }-d_{\min }}{\delta}$. Finally, let us select a generator $g_{0}$ for the group $\mathbb{Z}_{p_{0}}^{*}$. (E.g., in sensing the temperature of the atmosphere, it is enough to set $d_{\min }=-50$, $d_{\max }=50$ and $\delta=0.01$. Now, suppose that the number of involved sensors is no more than $N=20000$. Then, we can set $t=3, \kappa=17$ and then $p_{0}=2 \cdot 3^{17}=258280327$ and $g_{0}=5$.)

- Encryption. Each leaf sensor node $s_{i}$ encrypts the corresponding data $m_{i}$ by using the algorithm $c^{(i)}=\mathcal{E}_{+}\left(y ; m_{i}\right)$ and then sends $c^{(i)}$ to its parent sensor node.

- Aggregation.Each intermediate sensor node, $s_{j}$, collects all ciphertexts from its children nodes, $s_{j_{1}}, \cdots, s_{j_{r}}$ w.l.o.g., and then aggregates them by

$$
c^{(j)}=c^{\left(j_{1}\right)} \odot c^{\left(j_{2}\right)} \odot \cdots \odot c^{\left(j_{r}\right)} \odot \mathcal{E}_{+}\left(y ; m_{j}\right),
$$

where $m_{j}$ is the vaule measured by $s_{j}$ itself. Then, $s_{j} \operatorname{transmits} c^{(j)}$ to its parent sensor node, where similar aggregation is performed.

- Decryption.Finally, suppose that the sink node, say $s_{0}$, collects all ciphertexts from its children sensor nodes.

(1) At first, $s_{0}$ performs the aggregation operation in an identical manner to the general intermediate sensor nodes and obtains the ciphertext $c^{(0)}=$ $\left(c_{1}^{(0)}, c_{2}^{(0)}\right)$.

(2) Then, $s_{0}$ extracts the aggregated data $m$ by performing the decryption

$$
m=\mathcal{D}_{+}\left(x ; c^{(0)}\right) .
$$

Clearly, $m=\sum_{i} m_{i}$ holds. (Note that, since $N / \delta<p_{0}-1$, thus the formula (13) holds exactly even without modular operation. This condition is crucial for ensuring the correctness of summation obtained by using our additively homomorphic encryption scheme ${ }^{+}$HomElG.)

Additive aggregation can also be used to compute variance, standard deviation, and any other moments of the measured data [CMT05] even when the difference between the value obtained at each sensor node and the average of all values

${ }^{2}$ We can make a rough estimation on $N$ since our protocol is not sensitive to this boundary. 
obtained at the sensor nodes is unknown. Of course, for this purpose, the above aggregation protocol should be updated accordingly. For example, when computing the variance, each leaf sensor node computes and transmits $\mathcal{E}_{+}\left(y ; m_{i}\right)$ as well as $\mathcal{E}_{+}\left(y ; m_{i}^{2}\right)$ (i.e., the ciphertext of the square of $\left.m_{i}\right)$ to its parent sensor node, which in turn aggregates not only $\widehat{S^{(1)}}=\bigodot_{i} \mathcal{E}_{+}\left(y ; m_{i}\right)=\mathcal{E}_{+}\left(y ; \sum_{i=1}^{n} m_{i}\right)$ but also $\widehat{S^{(2)}}=\bigodot_{i} \mathcal{E}_{+}\left(y ; m_{i}^{2}\right)=\mathcal{E}_{+}\left(y ; \sum_{i=1}^{n} m_{i}^{2}\right)$. Eventually, the sink obtains

$$
D M=E M^{2}-(E M)^{2}=1 / n \cdot \mathcal{D}_{+}\left(x ; \widehat{S^{(2)}}\right)-\left(1 / n \cdot \mathcal{D}_{+}\left(x ; \widehat{S^{(1)}}\right)\right)^{2}
$$

where $D M$ and $E M$ indicate the variance and the expectation of the random variable $M$ defined over the message space $\mathcal{M}$, which should be re-defined as

$$
\mathcal{M}=\left\{0, \cdots,\left\lfloor\sqrt{\left(p_{0}-2\right) / N}\right\rfloor\right\}
$$

so as to let the potential maximal value of $\sum_{i=1}^{n} m_{i}^{2}$ lie in the set of $\left\{0, \cdots, p_{0}-\right.$ $2\}$. This also means we should select larger $p_{0}$ if it is not convenient to compress $\mathcal{M}$. Similarly, for computing $k(>2)$-order moments, even large primes $p_{0}$ should be used. Here, $n$ indicates the total number of sensor nodes involved in measuring data. We assume that intermediate sensor nodes also measure the relevant statistical data. Otherwise, $n$ should be the total number of leaf nodes. A general approach for calculating the appropriate $n$ is to view $n$ as a new statistical variable and count it during the process of counting $\widehat{S^{(1)}}$ and $\widehat{S^{(2)}}$. We can even maintain the anonymity of the sensor nodes involved in the measurement by employing the above protocol for the statistical variable $n$, i.e., by counting $n$ secretly. This task is essentially equivalent to e-voting, which can be implemented easily by an additively homomorphic encryption scheme.

In addition, by adopting the idea of Yokoo and Suzuki [21], it is not difficult to find the maximum of some secret numbers by using the proposed scheme.

\section{Acknowledgements}

We thank the anonymous referees for very useful comments. This work is supported by Japan NICT International Exchange Program (No. 2009-002), China National Natural Science Foundation Programs (No.90718001, No. 60973159), China 973 Program (No. 2007CB310704) and 863 Program (No. 2009AA012439), and Communicate University of China 211-project.

\section{References}

1. Bellare, M., Desai, A., Pointcheval, D., Rogaway, P.: Relations among notions of security for public-key encryption schemes. In: Krawczyk, H. (ed.) CRYPTO 1998. LNCS, vol. 1462, pp. 26-45. Springer, Heidelberg (1998)

2. Benaloh, J.: Dense probabilistic encryption. In: First Annual Workshop on Selected Areas in Cryptography, Kingston, ON, May 1994, pp. 120-128 (1994) 
3. Castelluccia, C., Mykletun, E., Tsudik, G.: Efficient aggregation of encrypted data in wireless sensor networks. In: MobiQuitous, pp. 109-117. IEEE Computer Society, Los Alamitos (2005)

4. Chevallier-Mames, B., Paillier, P., Pointcheval, D.: Encoding-free elgamal encryption without random oracles. In: Yung, M., Dodis, Y., Kiayias, A., Malkin, T.G. (eds.) PKC 2006. LNCS, vol. 3958, pp. 91-104. Springer, Heidelberg (2006)

5. Damgard, I., Geisler, M., Kroigard, M.: Homomorphic encryption and secure comparison. International Journal of Applied Cryptography (IJACT) 1(1), 22-31 (2008)

6. Hoogh, S.D., Schoenmakers, B., Skoric, B., Villegas, J.: Verifiable rotation of homomorphic encryptions. In: Jarecki, S., Tsudik, G. (eds.) PKC 2009. LNCS, vol. 5443, pp. 393-410. Springer, Heidelberg (2009)

7. Diffie, W., Hellman, M.E.: New directions in cryptography. IEEE Transactions on Information Theory 22(5), 644-654 (1976)

8. Dolev, D., Dwork, C., Naor, M.: Non-malleable cryptography (extended abstract). In: Proceedings of the 23rd Annual ACM Symposium on Theory of Computing (STOC 1991), pp. 542-552. ACM Press, New Orleans (1991)

9. ElGamal, T.: A public key cryptosystem and a signature scheme based on discrete logarithms. IEEE Transactions on Information Theory (TIT) 31(4), 469-472 (1985)

10. Ertaul, L., Kedlaya, V.: Computing aggregation function minimum/maximum using homomorphic encryption schemes in wireless sensor networks (WSNs). In: Arabnia, H.R., Clincy, V.A., Yang, L.T. (eds.) ICWN 2007, pp. 186-192. CSREA Press (2007)

11. Gentry, C.: On homomorphic encryption over circuits of arbitrary depth. In: Proceedings of the 41st Annual ACM Symposium on Theory of Computing (STOC 2009), pp. 169-178. ACM Press, Stanford University (2009)

12. Goldwasser, S., Micali, S.: Probabilistic encryption. J. Comput. Syst. Sci. (JCSS) 28(2), 270-299 (1984)

13. Goldwasser, S., Micali, S.: Probabilistic encryption and how to play mental poker keeping secret all partial information. In: Proceedings of the 14th Annual ACM Symposium on Theory of Computing (STOC 1982), pp. 365-377. ACM Press, San Francisco (1982)

14. Naor, M., Yung, M.: Public-key cryptosystems provably secure against chosen cypher-text attack. In: Proceedings of the 22nd Annual ACM Symposium on Theory of Computing (STOC 1990), pp. 427-437. ACM Press, Baltimore (1990)

15. Paillier, P.: Public-key cryptosystems based on composite degree residuosity classes. In: Stern, J. (ed.) EUROCRYPT 1999. LNCS, vol. 1592, pp. 223-238. Springer, Heidelberg (1999)

16. Rackoff, C., Simon, D.R.: Non-interactive zero-knowledge proof of knowledge and chosen ciphertext attack. In: Feigenbaum, J. (ed.) CRYPTO 1991. LNCS, vol. 576, pp. 433-444. Springer, Heidelberg (1992)

17. Shor, P.: Polynomail-time algorithms for prime factorization and discrete logarithms on a quantum computer. SIAM J. Comput. 5, 1484-1509 (1997)

18. Shoup, V.: A Computational Introduction to Number Theory and Algebra. Cambridge University Press, Cambridge (2005)

19. Ugus, O., Westhoff, D., Laue, R., Shoufan, A., Huss, S.A.: Optimized implementation of elliptic curve based additive homomorphic encryption for wireless sensor networks, CoRR abs/0903.3900 (2009)

20. Yacoub, H., Sarkar, T.K.: A homomorphic approach for through-wall sensing. IEEE Trans. Geoscience and Remote Sensing 47(5), 1318-1327 (2009) 
21. Yokoo, M., Suzuki, K.: Secure multi-agent dynamic programming based on homomorphic encryption and its application to combinatorial auctions. In: AAMAS 2002, pp. 112-119. ACM Press, New York (2002)

\section{Appendix A: Proof of Theorem 1}

Proof. According to the definition of the encryption and decryption, we have

$$
\begin{aligned}
& \mathcal{E}_{+}\left(y ; m_{1}\right) \odot \mathcal{E}_{+}\left(y ; m_{2}\right) \\
= & \left(g^{r_{1}}, y^{r_{1}} g_{0}^{m_{1}}\right) \odot\left(g^{r_{1}}, y^{r_{1}} g_{0}^{m_{1}}\right) \\
= & \left(g^{r_{1}+r_{2}}, y^{r_{1}+r_{2}} g_{0}^{m_{1}+m_{2}}\right) \\
= & \mathcal{E}_{+}\left(y ; m_{1}+m_{2}\right),
\end{aligned}
$$

(under new random number $r=r_{1}+r_{2}$ )

while

$$
\begin{aligned}
& \mathcal{D}_{+}\left(x ;\left(c_{11}, c_{12}\right)\right)+\mathcal{D}_{+}\left(x ;\left(c_{21}, c_{22}\right)\right) \\
\equiv & L_{g_{0}}\left(\mathcal{D}_{\times}\left(x ;\left(c_{11}, c_{12}\right)\right)+L_{g_{0}}\left(\mathcal{D}_{\times}\left(x ;\left(c_{21}, c_{22}\right)\right)\right.\right. \\
\equiv & L_{g_{0}}\left(c_{12} / c_{11}^{x}\right)+L_{g_{0}}\left(c_{22} / c_{21}^{x}\right) \\
\equiv & L_{g_{0}}\left(\frac{y^{r_{1}} \cdot g_{0}^{m_{1}}}{\left(g^{r_{1}}\right)^{x}}\right)+L_{g_{0}}\left(\frac{y^{r_{2}} \cdot g_{0}^{m_{2}}}{\left(g^{r_{2}}\right)^{x}}\right) \\
\equiv & L_{g_{0}}\left(\frac{\left(g^{x}\right)^{r_{1}} \cdot g_{0}^{m_{1}}}{\left(g^{r_{1}}\right)^{x}}\right)+L_{g_{0}}\left(\frac{\left(g^{x}\right)^{r_{2}} \cdot g_{0}^{m_{2}}}{\left(g^{r_{2}}\right)^{x}}\right) \\
\equiv & L_{g_{0}}\left(g_{0}^{m_{1}}\right)+L_{g_{0}}\left(g_{0}^{m_{2}}\right) \\
\equiv & m_{1}+m_{2}\left(\bmod p_{0}-1\right),
\end{aligned}
$$

and

$$
\begin{aligned}
& \mathcal{D}_{+}\left(x ;\left(c_{11}, c_{12}\right) \odot\left(c_{21}, c_{22}\right)\right) \\
\equiv & \mathcal{D}_{+}\left(x ;\left(g^{r_{1}}, y^{r_{1}} g_{0}^{m_{1}}\right) \odot\left(g^{r_{1}}, y^{r_{1}} g_{0}^{m_{1}}\right)\right) \\
\equiv & \mathcal{D}_{+}\left(x ;\left(g^{r_{1}+r_{2}}, y^{r_{1}+r_{2}} g_{0}^{m_{1}+m_{2}}\right)\right) \\
\equiv & L_{g_{0}}\left(\mathcal{D}_{\times}\left(x ;\left(g^{r_{1}+r_{2}}, y^{r_{1}+r_{2}} g_{0}^{m_{1}+m_{2}}\right)\right)\right) \\
\equiv & L_{g_{0}}\left(\frac{y^{r_{1}+r_{2}} g_{0}^{m_{1}+m_{2}}}{\left(g^{r_{1}+r_{2}}\right)^{x}}\right) \\
\equiv & L_{g_{0}}\left(\frac{\left(g^{x}\right)^{r_{1}+r_{2}} g_{0}^{m_{1}+m_{2}}}{\left(g^{r_{1}+r_{2}}\right)^{x}}\right) \\
\equiv & L_{g_{0}}\left(g_{0}^{m_{1}+m_{2}}\right) \\
\equiv & m_{1}+m_{2} \quad\left(\bmod p_{0}-1\right) .
\end{aligned}
$$

This concludes the theorem. 